Caries Res. 1968;2:355-356

\title{
Subject Index Vol. 2
}

Bacterial enzymes and dental plaque formation, 38 Bacterial polysaccharides, in caries etiology, 164 Bicarbonate-phosphate additives, effect on plaque $\mathrm{pH}, 27 \mathrm{Blood}$ serum, fluoride determination, 69 Bone-meal, effect on hamster caries, 338 -, reducing animal caries, 229

Calcification, of plaque, 19 Calcifying solution, effect on appearance of enamel caries, 87 Carbohydrate, oral clearance, 214 Carbonate, distribution in enamel, 1 Caries, at different ages, 79 -, in animals, and bone meal, 229 -, effect of saliva and calcifying solutions on histologic appearance, 87 -, in gnotobiotic animals, 139 -, in hamsters, influence of bone meal, $338-$, pattern of enamel demineralization, 180 -, prevalence correlations, 79 -, possible preventive effect of molybdenum, 262 Caustic lye stenosis of oesophagus, long-term effect on teeth, 347 Clearance of oral carbohydrates, 214

Demineralization, in enamel caries, 180 Diabetes, maternal, and changes

of primary teeth, 333

Enamel, acquired integuments, comparative analytical studies, 294 -, carbonate distribution, 1 Enamel, nature and importance of organic deposits, 104 -, pattern of carious demineralization, 180 phosphate, liberation by enzymes, 317 protein, histologic appearance and chemical composition, 281 structure, studies by microradio-graphy and two-dimensional microdensitometry, 235

Enzymes, liberating phosphate from enamel, 317

Fermentation, of glucose and sucrose by plaque streptococci, 193

Fluoridation, of water, in German Democratic Republic, 172

Fluoride, determination in blood serum, 69

-, effect of water intake on skeletal incorporation, 253

-, possible effect of molybdenum on distribution, 262

Glycoprotein, in saliva, 306 Gold plate technique, caries inhibition tests, 57

Hamster caries, influence of bone meal, 338

Immuno-fluorescence, in identification of plaque microorganisms, 273 356 


\section{Subject Index}

Integuments of enamel, comparative analytical studies, 294

Laser rays, effects on teeth, 10

Micro densitometry, two-dimensional, of enamel structure, 235

Microradiography, of enamel structure, 235

Micro-organisms, filamentous, in plaque, identification by immuno-fluorescence, 273

Molybdenum, placental transfer and possible caries-preventive effect, 262

Oesophageal stenosis from caustic lye, long term effect on teeth, 347 Oral carbohydrate

clearance, 214 Organic deposits, on enamel 104

Phosphate, liberation from enamel

by enzymes, 317 Plaque, 97 -, calcification, 19 -, effect on $\mathrm{pH}$ by sucrose and

bicarbonate-phosphate

additives, 27 -, formation as influenced by

bacterial enzymes, 38 -, histology and histochemistry, 115

- microorganisms, identification

by immuno-fluorescence, 273

-, mode of formation, 130

- $\quad$ streptococci, 147

sucrose, 193

, as fermentors of glucose and

, synthesising intracellular

polysaccharides, 201 Polysaccharides, bacterial, in caries etiology, 164

Polysaccharide, iodophilic, 47 Polysaccharides, in plaque

streptococci, 201 Polysaccharide, synthesis by

Streptococcus salivarius, 47 Primary teeth, changes in

maternal diabetes, 333 Protein, of enamel, histologic

appearance and chemical

composition, 281

Saliva, effect on appearance of

enamel caries, 87 -, glycoprotein components, 306 Scanning electron microscopy, of laser-exposed teeth, 10 Skeleton, of mice, effect of water

intake on fluoride incorporation

253 Sodium hexafluorostannate, tests

on caries inhibition, 57 Stenosis of oesophagus from

caustic lye, long-term effect

on teeth, 347 Streptococci, from plaque, as

fermentors of glucose and

sucrose, 193 -, of plaques, 147 -, synthesising intracellular

polysaccharides, $201-, \alpha$-haemolytic from plaque,

fermentation capacity, 193 Streptococcus salivarius, synthesis

of iodophilic polysaccharide, 47

Water fluoridation, in German Democratic Republic, 172

Water intake, effect on skeletal incorporation of fluoride, 253

X-ray crystallography, of laser-exposed teeth, 10 